\title{
Accelerated Hypofractionated Radiotherapy and Concurrent Etoposide/Cisplatin in Patients with Limited-Disease SCLC (LD-SCLC)
}

\author{
Wessam Elghamry, Ali Azmy, Iman Fouad, Zeinab Elsayed, Sherif Abdelwahab* \\ Department of Clinical Oncology, Ain Shams University, Cairo, Egypt \\ Email: *sherifok69@hotmail.com
}

How to cite this paper: Elghamry, W., Azmy, A., Fouad, I., Elsayed, Z. and Abdelwahab, S. (2020) Accelerated Hypofractionated Radiotherapy and Concurrent Etoposide/Cisplatin in Patients with Limited-Disease SCLC (LD-SCLC). Journal of Cancer Therapy, 11, 683-694. https://doi.org/10.4236/jct.2020.1111058

Received: September 25, 2020 Accepted: November 15, 2020 Published: November 18, 2020

Copyright $\odot 2020$ by author(s) and Scientific Research Publishing Inc. This work is licensed under the Creative Commons Attribution International License (CC BY 4.0).

http://creativecommons.org/licenses/by/4.0/

\begin{abstract}
Background: The optimal TRT dose/fraction for LD-SCLC remains debatable, and due to increasing number of population in Egypt and number of patients as well, so reducing the duration of radiation therapy is favored. This study was conducted using etoposide and cisplatin (EP) concurrently with accelerated hypofractionated TRT to evaluate the response and toxicity of this protocol in the treatment of patients with limited-disease small cell lung cancer (LD-SCLC). Patients and Methods: Thirty patients with previously untreated LD-SCLC were enrolled into this study between June 2012 and February 2015. All patients received etoposide $100 \mathrm{mg} / \mathrm{m}^{2}$ days 1 to 3 and cisplatin $25 \mathrm{mg} / \mathrm{m}^{2}$ days 1 to 3 with start of accelerated hypofractionated thoracic radiation therapy on first day of the second cycle of chemotherapy of $55 \mathrm{~Gy}$, $2.5 \mathrm{~Gy} /$ fraction over 30 days. Chemotherapy was given $4-6$ cycles. Prophylactic cranial irradiation $25 \mathrm{~Gy} / 10$ fractions were given for patients who achieved complete remission. Results: The median age was 60 years; 28 patients (93\%) were men. ECOG PS was 0 in $5(17 \%)$ patients and 1 in $12(40 \%)$ patients. Four (13\%) patients achieved a complete response (CR), 17 (57\%) patients achieved a partial response (PR), while 7 patients (23\%) had progressive disease (PD), and the ORR was $90 \%$. The median survival time was 26.4 months. The median PFS was 16.7 months. Among the hematologic toxicities neutropenia was the most prevalent toxicity and it was evident as grade 3 - 4 in $12(40 \%)$ patients. Grade 3 - 4 Asthenia was the most prevalent nonhematological toxicity, in 12 (40\%) patients; esophagitis occurred in 7 (23\%) patients. No treatment-related deaths (due to sepsis or bleeding) were reported in the study. Conclusion: Using etoposide and cisplatin concurrently with accelerated hypofractionated thoracic radiation therapy for the treatment of patients with LD-SCLC showed an encouraging outcome and acceptable toxicity and warrants further research.
\end{abstract}


Keywords

LD-SCLC, Etoposide, Cisplatin, Accelerated Radiation Therapy

\section{Introduction}

Small cell lung cancer (SCLC) is known to be a rapidly proliferating tumor having a tendency to metastasize early and widely. One third of patients with SCLC will present with limited disease SCLC (LD-SCLC), which is defined as disease confined to one hemithorax and which can be encompassed within a radiotherapy field of acceptable size [1] [2]. Currently, the standard of care for treating LD-SCLC is concurrent chemotherapy and thoracic radiation therapy (TRT), followed by prophylactic cranial irradiation (PCI) for the group of patients who achieve a good response after combined chemoradiotherapy, which has yielded a median survival of 15 to 23 months and 5-year survival rate up to 26\% [3] [4]. The optimal TRT dose/fraction for LD-SCLC remains debatable [3]. Intergroup study 0096 investigated once-daily and twice-daily TRT of 45 Gy in LD-SCLC, based on 2-dimensional radiation techniques, and the results showed that survival was improved significantly in the arm of twice daily TRT over 3 weeks, which has become one of the standard treatments [4]. However, despite using twice-daily TRT, there was a high local recurrence rate of $36 \%$ observed, which suggests that more intensified TRT should be considered for LD-SCLC. With more advanced radiation planning techniques, Cancer and Leukemia Group B carried out a series of trials using daily TRT with a high dosage of 70 Gy over 7 weeks in LD-SCLC [5] [6] [7]. In a trial done by Miller et al., complete response (CR) was achieved in $43 \%$ of included patients (95\% confidence interval [CI] $30 \%-56 \%$ ) and 38\% achieved partial response (PR). Median PFS was 12 months (95\% CI, 9 - 15 months) and median OS was 20 months (95\% CI, 16 - 24 months) [6], while in another trial done by Kelley et al., 7\% of the patients achieved CR and $64 \%$ of the patients achieved PR (response rate $71 \%, 95 \%$ confidence interval [CI], 59\% - 81\%) [7]. A pooled analysis of LD-SCLC Patients treated with induction chemotherapy followed by concurrent platinum-based chemotherapy and 70 Gy daily radiotherapy CALGB 30904 done by Salama et al. reported that there was no significant improvement of treatment outcome, this might be attributed to the prolonged overall radiation time [8]. It is known that accelerated repopulation of tumor cells during radiation therapy has shown negative effects in many tumor types and it is considered as one mechanism of resistance to treatment clinically [9] [10]. As SCLC has the characteristic of rapid doubling time and high growth fraction, there is also evidence suggesting that prolonged or interrupted overall radiation time contributes to treatment failure and poor outcome because of accelerated repopulation [11] [12] [13]. Xia et al. [14] found that overall radiation time might play an important role in the treatment of LD-SCLC and that patients treated with a high biologically effective 
dose (BED, including time factor) of $>57 \mathrm{~Gy}$ have favorable local control and survival [14]. A study conducted by Schild et al. [15] investigated the relationship between 5-year survival and various dose-fractionation regimens used in phase 3 trials reported between 1997 and 2004. A strong positive correlation between BED and 5-year survival was found in LD-SCLC (Pearson correlation coefficient 0.81 ). In order to obtain a higher intensive TRT regimen, beside the dose escalation there's is an alternative strategy which is the use of hypofractionated thoracic radiation therapy (HypoTRT). In the era of 2-dimensional radiation therapy, HypoTRT has been used as a safe and effective treatment for LD-SCLC in Canada (40 Gy/15 fractions) [16] [17]. Murray et al. reported that the median survival time and 5-year survival rate were 21.2 months and $22 \%$ respectively [16]; but, the cumulative risk for local recurrence exceeded 50\% beyond 3 years, which was partially attributed to the low radiation dose. There were much concerns that a fraction dose of $>2$ Gy may cause serious side effects and due to the limitation of 2-dimensional radiation techniques, the pace of exploring HypoTRT in LS-SCLC has slowed over the past few decades [18]. With the advances of 3-dimensional conformal radiation techniques (3D-CRT), a phase 1 study was conducted to determine the maximal tolerated dose of $\mathrm{Hy}$ poTRT for LD-SCLC [19]. Acute esophagitis was the predominant dose-limiting toxicity, and a dose between 50 Gy and 58 Gy was recommended. Based on the above-mentioned data, we conducted the present study using etoposide and cisplatin (EP) given concurrently with accelerated hypofractionated TRT to evaluate the response and toxicity of this protocol in the treatment of patients with limited-disease small cell lung cancer (LD-SCLC).

\section{Patients and Methods}

\subsection{Eligibility Criteria for Study Entry Included}

1) Patients must provide informed oral and/or written consent after approval of the local ethics committee 2) Patients have histologically or cytologically documented LD-SCLC, which was defined as disease confined to one hemithorax including bilateral supraclavicular nodes; other eligibility criteria were: 3) measurable disease, 4 ) age $<75$ years, 5) no previous treatment (neither chemotherapy nor radiotherapy), 6) Eastern Cooperative Oncology Group (ECOG) performance status of $\leq 2,7$ ) life expectancy of $>3$ months, 8) leucocyte count $\geq$ $4000 / \mathrm{mm}^{3}$, 9) platelet count $\geq 100,000 / \mathrm{mm}^{3}, 10$ ) hemoglobin $>9 \mathrm{~g} / \mathrm{dl}, 11$ ) serum creatinine $<1.4 \mathrm{mg} / \mathrm{dl}, 12$ ) creatinine clearance $\geq 60 \mathrm{ml} / \mathrm{min}, 13$ ) serum bilirubin $\leq 1.5 \mathrm{mg} / \mathrm{dl}, 14$ ) serum transaminase $<2 \times$ upper limit of normal (ULN), and 15) a life expectancy $\geq$ three months.

\subsection{Exclusion Criteria Included}

1) active severe infection, 2) severe heart disease, 3) malignant pleural or pericardial effusion, 4) chronic diarrhea, 5) intestinal obstruction or paralysis, and 6) active concomitant malignancy. Pregnant or lactating women were also excluded. 


\subsection{Pretreatment Assessment and Post-Treatment Reassessment}

Each patient must have had the following assessment tests before being enrolled in the study: full medical history and clinical examination; baseline tests including a full blood count, serum biochemistry (urea and electrolytes, liver function tests, calcium, and lactate dehydrogenase $[\mathrm{LDH}]$ ) and a chest X-ray. Staging procedure for all patients included computed tomography (CT) scan of the chest and upper abdomen with contrast. CT scan and/or magnetic resonance imaging (MRI) of brain or a bone scan was not routinely required. PET/CT scan was performed for some cases. Pulmonary function tests were also used (including forced expiratory volume in the first second [FEV1], forced vital capacity, and arterial blood gases). Patients were staged according to the Veterans' Administration Lung Cancer Study Group as having limited disease (confined to one hemithorax including contralateral mediastinal and supraclavicular nodes). Patients were followed after completion of the course of treatment every 2 months until disease progression or death. Radiological responses were documented by a CT scan of the chest after 4 weeks. Treatment toxicity was classified according to the criteria of the World Health Organization [20], except for radiation-induced esophagitis, for which we used the ECOG criteria [21] while pneumonitis was clinically and radiographically graded according to the Radiation Therapy Oncology Group (RTOG) acute and late lung morbidity scoring criteria [22]. Tumor response was evaluated after every two cycles of chemotherapy using the same evaluation method and it was classified according to the WHO criteria. A complete response (CR) was defined as the disappearance of any evidence of tumors for at least 4 weeks. A partial response (PR) was defined as $\geq 50 \%$ reduction in the sum of the products of the greatest perpendicular diameters of all lesions for at least 4 weeks. Stable disease (SD) was defined as $<50 \%$ reduction or $<25 \%$ increase in the products of the greatest perpendicular diameters of all lesions without any evidence of new lesions. Progressive disease (PD) was defined as an increase of $\geq 25 \%$ or the appearance of new lesions.

\subsection{Chemotherapy}

Chemotherapy was given a 21-day interval for 4 - 6 cycles, however, the interval was extended to 28 days during concurrent TRT. The chemotherapy regimen (etoposide/cisplatin [EP]) consisted of cisplatin $25 \mathrm{mg} / \mathrm{m}^{2}$ per day administered on days 1 to 3 and etoposide $100 \mathrm{mg} / \mathrm{m}^{2}$ per day on days 1 to 3 . After 4 cycles of EP chemotherapy, patients with good performance status $(0-2)$ and without complete response, continued to receive chemotherapy to a total of 6 cycles. The application of granulocyte colony stimulating factor (G-CSF) was allowed when the absolute granulocyte count was $<1000 / \mathrm{mm}^{3}$. Doses were modified on the basis of blood counts, serum chemistry values, and toxicity levels. Chemotherapy was discontinued for patients with two dose reductions. 


\subsection{Thoracic Radiation Therapy (TRT)}

HypoTRT was initiated at the first day of the second cycle of chemotherapy. An enhanced CT scan of the thorax was performed to help in radiation therapy planning. Gross target volume was based on the restaging chest CT obtained after induction chemotherapy, including the residual primary tumor and lymph nodes $>1 \mathrm{~cm}$ in short axis diameter observed on initial thoracic CT scans. If the lymph nodes became smaller or disappeared after induction chemotherapy, the previously involved lymph nodal regions were included in the radiation target according to the prechemotherapy CT scans. The planning target volume (PTV) included the primary lesion (gross tumor volume) with a 1- to $1.5-\mathrm{cm}$ margin in all directions. No elective nodal irradiation was carried out. HypoTRT with a total dose of 55 Gy was administered daily at 2.5 Gy per fraction over 30 days. Three to four beams with a 6- or 10-MV photon were used, and the dose constraints to the surrounding normal organs were as follows: maximum spinal cord dose $\leq 42 \mathrm{~Gy}$; mean lung dose $\leq 15 \mathrm{~Gy}$, and V20 (percentage of total lung volume receiving $>20 \mathrm{~Gy}$ ) $\leq 25 \%$ (for the calculation of mean lung dose and V20, the volume of both lungs minus the PTV was used); mean esophagus dose $\leq 34 \mathrm{~Gy}$; and mean heart dose $\leq 30 \mathrm{~Gy}$. Dose distributions to normal tissue < $10 \%$ of the upper limitation were considered as acceptable variation. Any interruption or delay was discouraged during TRT unless any grade 4 hematologic toxicity (including absolute neutrophil count $\leq 1000 / \mathrm{mm}^{3}$, platelet count $\leq$ $50,000 / \mathrm{mm}^{3}$ ), febrile neutropenia, or grade $\geq 3$ esophagitis or pneumonitis occurred.

\subsection{Prophylactic Cranial Irradiation (PCI)}

Patients who achieved a complete response (CR) had received PCI which started 5 weeks after the end of the last course of chemotherapy. A total dose of $30 \mathrm{~Gy}$ was administered in 10 fractions of $3 \mathrm{~Gy}$ (5 fractions/weeks).

\subsection{Statistics}

The primary objective of this study was to evaluate the response and toxicity of an EP regimen concurrent with Accelerated Hypo-TRT for LD-SCLC patients. Any patient who received at least one cycle of chemotherapy was considered assessable for toxicity, and all eligible patients who received even one cycle of chemotherapy were included for survival estimation. Overall survival (OS) was measured from the date of the first chemotherapy administration to the date of death or last follow-up visit. Progression-free survival (PFS) was measured from the date of the first administration of the chemotherapy up to the date of disease progression or death from any cause or the date of last follow-up visit. Overall survival (OS) and PFS were estimated using the Kaplan-Meier method [23]. The statistical analysis was carried out using SPSS software version 13 (SPSS, Chicago, IL, USA). 


\section{Results}

\subsection{Patient Characteristics}

Thirty patients with previously untreated LD-SCLC were enrolled into the study between June 2012 and February 2015. Table 1 summarizes the characteristics of these 30 patients.

\subsection{Response}

In the 30 patients assessed for response after the first cycle of chemotherapy and before starting TRT 4 (13\%) patients achieved a CR, 17 patients (57\%) achieved a PR, $3(10 \%)$ patients achieved SD while 7 patients (23\%) had PD; 6 (20\%) of the 10 non-responders achieved a PR after commencing concurrent chemoradiotherapy; therefore, the overall response rate was $90 \%$.

\subsection{Overall Survival (OS) and Progression-Free Survival (PFS)}

The median OS time was 26.4 months (95\% confidence interval [CI], 10.4 - 28.1 months), and 1-, and 2-years OS rates were $78 \%$ and $58.3 \%$, respectively (Figure 1). The median PFS was 16.7 months (95\% CI, 6.7 - 19.0 months), and 1-, and 2 -years PFS rates were 60\%, and $41.4 \%$, respectively (Figure 2).

\subsection{Treatment Toxicity}

Table 2 summarizes the incidence and the severity of the hematological and non-hematological toxicities as well. Neutropenia was reported to be the most prevalent hematological toxicity which occurred as a grade $3-4$ in 7 (23\%) patients, while asthenia was the most prevalent non-hematological toxicity which occurred as a grade 3 - 4 in 12 (40\%) patients. Fortunately, no treatment-related deaths (due to sepsis or bleeding) were reported in the study.

Table 1. Patient characteristics.

\begin{tabular}{ccc}
\hline & \multicolumn{2}{c}{ Patients } \\
\cline { 2 - 3 } Characteristic & No. & \\
\hline Age, years & & \\
Median & 60 & \\
Range & $49-71$ & \\
\hline Sex & & $93 \%$ \\
Male & 28 & $7 \%$ \\
Female & 2 & $17 \%$ \\
ECOG PS & & $40 \%$ \\
0 & 5 & $43 \%$ \\
\hline
\end{tabular}


overall survival

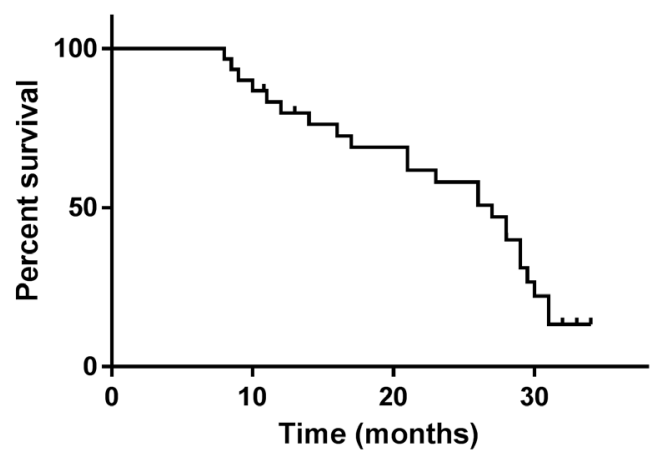

Figure 1. Overall survival.

PFS

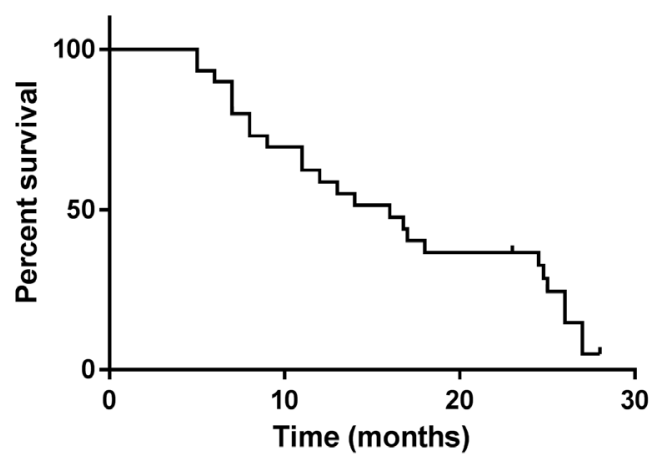

Figure 2. Progression-free survival.

Table 2. Treatment-related toxicity.

\begin{tabular}{|c|c|c|c|c|c|c|}
\hline \multirow[t]{2}{*}{ Toxicity } & \multicolumn{2}{|c|}{ Grade 2} & \multicolumn{2}{|c|}{ Grade 3} & \multicolumn{2}{|c|}{ Grade 4} \\
\hline & No. & $\%$ & No. & $\%$ & No. & $\%$ \\
\hline \multicolumn{7}{|l|}{ Hemaltological } \\
\hline Anemia & 7 & 23 & 8 & 27 & 1 & 3 \\
\hline Neutropenia & 5 & 17 & 15 & 30 & 1 & 3 \\
\hline Thrombocytopenia & 6 & 20 & 3 & 10 & 1 & 3 \\
\hline \multicolumn{7}{|l|}{ Non-hematological } \\
\hline Pneumonitis & 3 & 10 & 3 & 10 & 1 & 3 \\
\hline Esophagitis & 8 & 27 & 6 & 20 & 0 & 0 \\
\hline Asthenia & 11 & 37 & 7 & 23 & 1 & 3 \\
\hline Diarrhea & 4 & 13 & 2 & 7 & 0 & 0 \\
\hline Nausea & 8 & 27 & 6 & 20 & 2 & 7 \\
\hline Vomiting & 5 & 17 & 5 & 17 & 1 & 3 \\
\hline Infection & 4 & 13 & 2 & 7 & 0 & 0 \\
\hline Elevated S. creatinine & 3 & 10 & 3 & 10 & 0 & 0 \\
\hline
\end{tabular}




\subsection{Patterns of Failure}

Distant metastasis represented the most common pattern of failure. Five (17\%) patients developed locoregional recurrence, and 15 (50\%) patients were reported to have distant metastases. Second line chemotherapy in the form of Irinotecan/Platinum was given for those patients who developed PD or those who relapsed after being in remission.

\section{Discussion}

Combined modality therapy has emerged as the accepted standard of treatment for patients with LD-SCLC. However, neither an optimal radiotherapy dose nor an optimal sequencing schedule has been established [24]. The addition of TRT has improved the survival of LD-SCLC patients. It was previously demonstrated that TRT combined with EP is more effective for LD- SCLC compared to radiotherapy and the hematological toxicity was more severe in the concurrent arm [25]. A-Hypofractionated TRT schedules may confer a survival benefit compared with prolonged conventional fractionated TRT [4] and there is some evidence suggesting that an improvement of outcome in radiochemotherapy of LD-SCLC is related to treatment intensification by shortening the total duration of therapy [26]. The optimal timing of TRT related to chemotherapy is another important, yet still unresolved issue [27]. Early administration of a hypofractionated TRT may confer the benefit of avoiding excessive toxicity while maintaining high efficacy [28]. Because of increased toxicity and inconvenience the twice-daily TRT was not widely adopted, once-daily TRT remained the most commonly used regimen for LD-SCLC [29]. Once-daily Hypofracionated TRT with a shortening of the overall treatment time may optimize survival and tumor control [13]. A favorable efficacy of Hypofractionated TRT (40 Gy/15 F) early in 1993, during the era of two dimensional radiotherapies has been reported, with a median overall survival of 21.2 months and 5-year survival rate of $22 \%$. In our study we recruited 30 patients with LD-SCLC, $93 \%$ of the patients were men [16]. In the current study ORR was reported to be $90 \%$, while in a similar trial done by Zhang et al. in the HYPO-RT arm, ORR was $97 \%$ [30]. In the current study the median PFS was 16.7 months (95\% CI, 6.7 - 19.0 months), and 1-, and 2 -years PFS rates were $60 \%$, and $41.4 \%$, respectively, when compared to the study by Zhang et al. [30], where the median PFS was 18.2 months (95\% CI: 15.8 - 20.6 months), and the 1-year and 2-year PFS rates were $64.8 \%$ and $32.4 \%$, respectively. The median OS in our study was 26.4 months (95\% CI, $10.4-28.1$ months), and 1-, and 2-years OS rates were $78 \%$ and $58.3 \%$, compared to the results of the study by Zhang et al. [30], where the median OS was 27.2 months (95\% CI: 25.2 - 29.2 months), and the 1- and 2-year survival rates were $87.0 \%$ and $62.2 \%$, respectively.

As a natural behavior of SCLC, distant metastasis was reported to be the most common pattern of failure which occurred in $50 \%$ of the patients in our study, while $17 \%$ patients developed locoregional recurrence. These findings are almost 
the same like what was found in the study done by Zhang et al. [30], where locoregional recurrence occurred in $17.4 \%$ and distant metastasis occurred in $42 \%$ of the patients recruited into the study.

The toxicities in our study seemed tolerable and acceptable, grade 3 - 4 neutropenia occurred in $23 \%$ of patients, these findings are much less than the study done by Gronberg et al., where $86 \%$ of the patients in that study developed grade 3 - 4 neutropenia [31], while grade 3 - 4 esophagitis occurred in $20 \%$ of the patients in our study which is less than that reported in the study done by Gronberg et al. which was $31 \%$ [31]. Radiation-associated pneumonitis is always a problem facing treatment of such a disease, and we reported that grade $3-4$ pneumonitis occurred in $13 \%$ of the patients in the present study which was compared with $2 \%$ and $8.6 \%$ of the patients in the studies done by Gronberg et al. and Zhang et al. respectively [30] [31].

In conclusion, using etoposide and cisplatin, when given concurrently with accelerated hypofractionated TRT for the treatment of patients with LD-SCLC, showed an encouraging outcome and acceptable toxicity and warrants further research especially in centers that have a long waitlist.

\section{Conflict of Interest}

All authors indicated no potential conflict of interest including any financial, personal, or other relationships with other people or organizations that could inappropriately influence their work.

\section{References}

[1] Warde, P. and Payne, D. (1992) Does Thoracic Irradiation Improve Survival and Local Control in Limited-Stage Small-Cell Carcinoma of the Lung? A Meta-Analysis. Journal of Clinical Oncology, 10, 890-895. https://doi.org/10.1200/JCO.1992.10.6.890

[2] Pignon, J.P. and Arriagada, R. (1992) Role of Thoracic Radiotherapy in Limited-Stage Small-Cell Lung Cancer: Quantitative Review Based on the Literature versus Meta-Analysis Based on Individual Data. Journal of Clinical Oncology, 10, 1819-1820. https://doi.org/10.1200/JCO.1992.10.11.1819

[3] Kong, F.M., Lally, B.E., Chang, J.Y., et al. (2013) ACR Appropriateness Criteria. Radiation Therapy for Small-Cell Lung Cancer. American Journal of Clinical Oncology, 36, 206-213. https://doi.org/10.1097/COC.0b013e31827e5523

[4] Turrisi, A.T., Kim, K., Blum, R., et al. (1999) Twice-Daily Compared with Once-Daily Thoracic Radiotherapy in Limited Small-Cell Lung Cancer Treated Concurrently with Cisplatin and Etoposide. The New England Journal of Medicine, 340, 265-271. https://doi.org/10.1056/NEJM199901283400403

[5] Bogart, J.A., Herndon, J.E., Lyss, A.P., Lee, M.E., et al. (2004) 70 Gy thoracic Radiotherapy Is Feasible Concurrent with Chemotherapy for Limited-Stage Small-Cell Lung Cancer: Analysis of Cancer and Leukemia Group B Study 39808. International Journal of Radiation Oncology, Biology, Physics, 59, 460-468. https://doi.org/10.1016/j.ijrobp.2003.10.021

[6] Miller, A.A., Wang, X.F., Bogart, J.A., et al. (2007) Phase II Trial of Paclitaxel-Topotecan-Etoposide Followed by Consolidation Chemoradiotherapy for $\mathrm{Li}$ - 
mited-Stage Small Cell Lung Cancer: CALGB 30002. International Journal of Radiation Oncology, Biology, Physics, 2, 645-651.

https://doi.org/10.1097/JTO.0b013e318074bbf5

[7] Kelley, M., Bogart, J., Hodgeson, L., et al. (2013) Phase II Study of Induction Cisplatin and Irinotecan Followed by Concurrent Carboplatin, Etoposide, and Thoracic Radiotherapy for Limited Stage Small Cell Lung Cancer, CALGB 30206. Journal of Thoracic Oncology, 8, 102-108. https://doi.org/10.1097/JTO.0b013e31827628e1

[8] Salama, J.K., Hodgson, L., Pang, H., et al. (2013) A Pooled Analysis of Limited-Stage Small-Cell Lung Cancer Patients Treated with Induction Chemotherapy Followed by Concurrent Platinum-Based Chemotherapy and 70 Gy Daily Radiotherapy CALGB 30904. Journal of Thoracic Oncology, 8, 1043-1049. https://doi.org/10.1097/JTO.0b013e318293d8a4

[9] Overgaard, J., Hansen, H.S., Specht, L., et al. (2003) Five Compared with Six Fractions Per Week of Conventional Radiotherapy of Squamous-Cell Carcinoma of Head and Neck: DAHANCA 6 and 7 Randomized Controlled Trial. Lancet, 362, 933-940. https://doi.org/10.1016/S0140-6736(03)14361-9

[10] Bese, N.S., Hendry, J. and Jeremic, B. (2007) Effects of Prolongation of Overall Treatment Time Due to Unplanned Interruptions during Radiotherapy of Different Tumor Sites and Practical Methods for Compensation. International Journal of Radiation Oncology, Biology, Physics, 68, 654-661.

https://doi.org/10.1016/j.ijrobp.2007.03.010

[11] Schild, S.E., Bonner, J.A., Shanahan, T.G., et al. (2004) Long-Term Results of a Phase III Trial Comparing Once-Daily Radiotherapy with Twice-Daily Radiotherapy in Limited-Stage Small-Cell Lung Cancer. International Journal of Radiation Oncology, Biology, Physics, 59, 943-951. https://doi.org/10.1016/j.ijrobp.2004.01.055

[12] De Ruysscher, D., Pijls-Johannesma, M., Bentzen, SM., et al. (2006) Time between the First Day of Chemotherapy and the Last Day of Chest Radiation Is the Most Important Predictor of Survival in Limited-Disease Small Cell Lung Cancer. Journal of Clinical Oncology, 24, 1057-1063. https://doi.org/10.1200/JCO.2005.02.9793

[13] Videtic, G.M., Fung, K., Tomiak, A.T., et al. (2001) Using Treatment Interruptions to Palliate the Toxicity from Concurrent Chemoradiation for Limited Small Cell Lung Cancer Decreases Survival and Disease Control. Lung Cancer, 33, 249-258. https://doi.org/10.1016/S0169-5002(00)00240-3

[14] Xia, B., Chen, G.Y., Cai, X.W., et al. (2011) The Effect of Bioequivalent Radiation Dose on Survival of Patients with Limited-Stage Small-Cell Lung Cancer. Radiation Oncology, 6, Article No. 50. https://doi.org/10.1186/1748-717X-6-50

[15] Schild, S.E., Bonner, J.A., Hillman, S., et al. (2007) Results of a Phase II Study of High-Dose Thoracic Radiation Therapy with Concurrent Cisplatin and Etoposide in Limited-Stage Small-Cell Lung Cancer (NCCTG 95-20-53). Journal of Clinical Oncology, 25, 3124-3129. https://doi.org/10.1200/JCO.2006.09.9606

[16] Murray, N., Coy, P., Pater, J.L., Hodson, I., Arnold, A., Zee, B.C., Payne, D., Kostashuk, E.C., Evans, W.K. and Dixon, P. (1993) Importance of Timing for Thoracic Irradiation in the Combined Modality Treatment of Limited-Stage Small Cell Lung Cancer. The National Cancer Institute of Canada Clinical Trials Group. Journal of Clinical Oncology, 11, 336-344. https://doi.org/10.1200/JCO.1993.11.2.336

[17] Work, E., Nielsen. O.S., Bentzen, S.M., Fode, K. and Palshof, T. (1997) Randomized Study of Initial versus Late Chest Irradiation Combined with Chemotherapy in Limited Stage Small-Cell Lung Cancer. Aarhus Lung Cancer Group. Journal of Clini- 
cal Oncology, 15, 3030-3037. https://doi.org/10.1200/JCO.1997.15.9.3030

[18] Videtic, G.M., Truong, P.T., Dar, A.R., Yu, E.W. and Stitt, L.W. (2003) Shifting from Hypofractionated to "Conventionally" Fractionated Thoracic Radiotherapy: A Single Institution's 10-Year Experience in the Management of Limited-Stage Small-Cell Lung Cancer Using Concurrent Chemoradiation. International Journal of Radiation Oncology, Biology, Physics, 57, 709-716. https://doi.org/10.1016/S0360-3016(03)00635-7

[19] Yee, D., Halperin, R., Hanson, J., et al. (2006) Phase I Study of Hypofractionated Dose-Escalated Thoracic Radiotherapy for Limited-Stage Small-Cell Lung Cancer. International Journal of Radiation Oncology, Biology, Physics, 65, 466-473. https://doi.org/10.1016/j.ijrobp.2005.12.013

[20] World Health Organization (1979) Handbook for Reporting Results of Cancer Treatment. World Health Organization, Geneva.

[21] Oken, M.M., Creech, R.H., Tormey, D.C., et al. (1982) Toxicity and Response criteria of the Eastern Cooperative Oncology Group. American Journal of Clinical Oncology, 5, 649-655. https://doi.org/10.1097/00000421-198212000-00014

[22] Byhardt, R.W., Martin, L. and Pajak, T.F. (1993) The Influence of Field size and Other Treatment Factors on Pulmonary Toxicity Following Hyperfractionated Irradiation for Inoperable Non-Small-Cell Lung Cancer (NSCLC)-Analysis of a Radiation Therapy Oncology Group (RTOG) Protocol. International Journal of Radiation Oncology, Biology, Physics, 27, 537-544. https://doi.org/10.1016/0360-3016(93)90377-8

[23] Kaplan, E.L. and Meier, P. (1958) Non-Parametric Estimation from Incomplete Observation. Journal of the American Statistical Association, 53, 457-481. https://doi.org/10.1080/01621459.1958.10501452

[24] Abdelwahab, S., Abdulla, H., Azmy, A., et al. (2009) Integration of Irinotecan and Cisplatin with Early Concurrent Conventional Radiotherapy for Limited-Disease SCLC (LD-SCLC). International Journal of Clinical Oncology, 14, 230-236. https://doi.org/10.1007/s10147-008-0842-7

[25] Takada, M., Fukuoka, M., Kawahara, M., et al. (2002) Phase III Study of Concurrent versus Sequential Thoracic Radiotherapy in Combination with Cisplatin and Etoposide for Limited-Stage Small-Cell Lung Cancer: Results of the Japan Clinical Oncology Group Study 9104. Journal of Clinical Oncology, 20, 3054-3060. https://doi.org/10.1200/JCO.2002.12.071

[26] Pijls-Johannesma, M., De Ruysscher, D., Vansteenkiste, J., Kester, A., Rutten, I. and Lambin, P. (2007) Timing of Chest Radiotherapy in Patients with Limited Stage Small Cell Lung Cancer: A Systematic Review and Meta-Analysis of Randomised Controlled Trials. Cancer Treatment Reviews, 33, 461-473. https://doi.org/10.1016/j.ctrv.2007.03.002

[27] Früh, M., De Ruysscher, D., Popat, S., Crinò, L., Peters, S. and Felip, E. (2013) Small-cell Lung Cancer (SCLC): ESMO Clinical Practice Guide-lines for Diagnosis, Treatment and Follow-Up. Annals of Oncology, 24, vi99-vi105. https://doi.org/10.1093/annonc/mdt178

[28] Socha, J., Guzowska, A., Tyc-Szczepaniak, D., Wierzchowski, M., Sprawka, A., Szczesna, A. and Kepka, L. (2015) Accelerated Hypofractionated Thoracic Radiotherapy in Limited Disease Small Cell Lung Cancer: Comparison with the Results of Conventionally Fractionated Radiotherapy. JBUON, 20, 146-157.

[29] De Ruysscher, D. and Vansteenkiste, J. (2000) Chest Radiotherapy in Limited-Stage Small Cell Lung Cancer: Facts, Questions, Prospects. Radiotherapy \& Oncology, 55, 1-9. https://doi.org/10.1016/S0167-8140(00)00156-0 
[30] Zhang, J., Fan, M., Liu, D., Zhao, K.L., Wu, K.L., Zhao, W.X., Zhu, Z.F. and Fu, X.L. (2017) Hypo- or Conventionally Fractionated Radiotherapy Combined with Chemotherapy in Patients with Limited Stage Small Cell Lung Cancer. Radiation Oncology, 12, Article No. 51. https://doi.org/10.1186/s13014-017-0788-X

[31] Gronberg, B.H., Halvorsen, T.O., Flotten, O., et al. (2016) Randomized Phase II Trial Comparing Twice Daily Hyperfractionated with Once Daily Hypofractionated Thoracic Radiotherapy in Limited Disease Small Cell Lung Cancer. Acta Oncologica, 55, 591-597. https://doi.org/10.3109/0284186X.2015.1092584 